\title{
Robust Correction Procedure for Accurate Thin Shell Models via a Perturbation Technique
}

\author{
Vuong Dang Quoc \\ School of Electrical Engineering \\ Hanoi University of Science and Technology \\ Hanoi, Vietnam \\ vuong.dangquoc@hust.edu.vn
}

\begin{abstract}
This research proposes a robust correction procedure to improve inaccuracies around edges and corners inherent to thin shell electromagnetic problems by means of perturbation technique. This proposal is developed with three processes: A classical thin shell approximation replaced with an impedancetype interface condition across a surface is first considered and then a volume correction is introduced to overcome the thin shell approximation. However, the volume correction is quite sensitive to cancellation errors, with dramatic effects in the calculation of the local fields near edges and corners. Therefore, a robust correction procedure is added to improve cancellation errors of the volume correction. Each step of the developed method is validated on the practical problem.
\end{abstract}

Keywords-thin shell approximation; magnetic field; eddy current; joule power loss; perturbation method; subproblem method

\section{INTRODUCTION}

Thin Sell (TS) models [1-3] are approximated by a priori known 1-D analytical distributions to avoid meshing the thin regions and lighten the mesh of surrounding regions with Interface Conditions (ICs). However, these ICs generally neglect end and border effects, which lead to inaccuracies in the calculation of local and global fields in the vicinity of geometrical discontinuities near borders and corners, increasing with thickness. In order to scope with this difficulty, a sub problem technique has been implemented for correcting errors surrounding the corners and edges appearing from the TS [4-5, 11]. But in this development, the volume correction is quite sensitive to cancellation errors, with the calculation of local fields (magnetic fields, eddy current losses, and joule power losses) near edges and corners. In this study, a robust correction procedure is embedded in the subproblem technique to guarantee the obtained solutions from volume corrections without cancellation errors in magnetic materials. The method is extended in the hearth of the Sub Problem Method (SPM), allowing a complete solution to express as a sum of Sub Problems (SPs) solutions, already to applied numerous corrections and problem splitting [4-5]. The extensions are herein implemented for the magnetic vector potential Finite Element (FE) magnetodynamic formulation, paying special attention to the proper discretization of the constraints involved in each SP.

\section{SEQUENCE OF THE PERTURBATION TECHNIQUE}

\section{A. Definition of Coupled Sub Problems}

Based on the SPM strategy [3-5], the scenario of the perturbation method is herein considered in two steps: A problem attending with the stranded inductor and TS model is first solved on a simplified mesh. The inaccuracy on TS solution is then improved by the volume correction taken by a robust correction procedure in order to overcome the cancellation error [4-7]. The relationship between SPs is constrained by surface sources (SSs) or volume sources (VSs), where SSs show changes of ICs across surfaces from previous SPs, and VSs point out changes of material properties of volume thin regions. Each SP is directly performed on its own mesh without depending on other meshes or solving again a new full problem for each new set of parameters as a traditional finite element method $[7,8,10]$.

\section{B. Canonical Magnetodynamic Problem}

In the SP scenario, a canonical magnetodynamic problem $p$ is defined in a domain $\Omega_{i}$, with boundary $\partial \Omega_{p}=\Gamma_{p}=$ $\Gamma_{h, p} \cup \Gamma_{e, p}$. It should be noted that the subscript $p$ refers to the associated SP $p$. The eddy current conducting part of $\Omega_{p}$ is denoted $\Omega_{c, p}$ and the non-conducting one $\Omega_{c, p}^{C}$, with $\Omega_{p}=\Omega_{c, p} \cup \Omega_{c, p}^{C}$. Stranded inductors belong to $\Omega_{c, i}^{C}$. The equations, material relations, and boundary conditions (BCs) of the SP $p$ are [4-7]:

$$
\begin{aligned}
\operatorname{curl} \boldsymbol{h}_{p}=\boldsymbol{j}_{p}, \operatorname{div} \boldsymbol{b}_{p}=0, \text { curle } \boldsymbol{e}_{p}=-\partial_{t} \boldsymbol{b}_{p}, \quad(1 \mathrm{a}-\mathrm{b}-\mathrm{c}) \\
\boldsymbol{h}_{i}=\mu_{p}^{-1} \boldsymbol{b}_{p}+\boldsymbol{h}_{s, p}, \boldsymbol{j}_{p}=\sigma_{p} \boldsymbol{e}_{p}+\boldsymbol{j}_{s, p} \quad(2 \mathrm{a}-\mathrm{b}) \\
{\left[\boldsymbol{n} \times \boldsymbol{h}_{p}\right]_{\Gamma_{h, p}}=\boldsymbol{k}_{f, p}, \boldsymbol{n} \times\left.\boldsymbol{h}_{p}\right|_{\Gamma_{h, p}}=0 \quad(3 \mathrm{a}-\mathrm{b}) }
\end{aligned}
$$

where $\boldsymbol{h}_{p}$ is the magnetic field, $\boldsymbol{b}_{p}$ is the magnetic flux density, $\boldsymbol{e}_{p}$ is the electric field, $\boldsymbol{j}_{s, p}$ is the electric current density, $\mu_{p}$ is the magnetic permeability, $\sigma_{p}$ is the electric conductivity and $\boldsymbol{n}$ is the unit normal exterior to $\Omega_{p}$.

The source fields $\boldsymbol{h}_{s, p}$ and $\boldsymbol{j}_{s, p}$ in (2a-b) are VSs. In the frame of SPM, the field $\boldsymbol{h}_{s, p}$ is usually considered as a remnant field in magnetic materials and expressed changes of permeability. The field $\boldsymbol{j}_{s, p}$ is an imposed electric current 
density in inductors and presented changes of conductivity. For that, the changes from SP $u$ ( $\mu_{u}$ and $\sigma_{u}$ to SP $p\left(\mu_{p}\right.$ and $\left.\sigma_{p}\right)$, $\boldsymbol{h}_{s, p}$ and $\left.\boldsymbol{j}_{s, p}\right)$ are defined [4-7]:

$$
\boldsymbol{h}_{s, p}=\left(\mu_{p}^{-1}-\mu_{u}^{-1}\right) \boldsymbol{b}_{u}, \quad \boldsymbol{j}_{s, p}=\left(\sigma_{p}-\sigma_{u}\right) \boldsymbol{e}_{u}
$$

The total fields to be related by the uploaded relations $\boldsymbol{h}=\mu_{p}^{-1}\left(\boldsymbol{b}_{p}+\boldsymbol{b}_{u}\right)$ and $\boldsymbol{j}=\sigma_{p}\left(\boldsymbol{e}_{p}+\boldsymbol{e}_{u}\right)[3,4]$. The source field $\boldsymbol{k}_{f, p}$ in (3a) is SSs. In general, these SSs are defined as zero for classical homogeneous BCs. ICs can define their discontinuities through any interface $\gamma_{p}$ (with sides $\gamma_{p}^{+}$and $\gamma_{p}^{-}$) in $\Omega_{p}$, with the notation $[\cdot]_{\gamma_{p}}=\left.\right|_{\gamma_{p}^{+}}-\left.\right|_{\gamma_{p}^{-}}$. On the other hand, they can be considered as SSs for particular phenomena appearing at the both sides $\left(\gamma_{p}^{+}\right.$and $\left.\gamma_{p}^{-}\right)$of the thin regions [3, 4].

\section{Thin Shell Finite Element Model}

The constraints between the TS model and volume corrections are expressed via VSs and SSs. For the magnetic vector potential formulation ( $\boldsymbol{b}$-conformal formulation) in the TS model [3], these sources are defined via the BCs and ICs of impedance-type boundary conditions (IBC) associated with solutions from previous SP which can be a stranded inductor alone or the inductors with the TS model. Therefore, the constraint from the TS model to the volume correction problems, or from the volume corection to the robust correction procedure, can be obtained from [3], i.e.:

$$
\begin{gathered}
{\left[\boldsymbol{n} \times \boldsymbol{h}_{p}\right]_{\gamma_{p}}=-\sigma \beta \partial_{t}\left(2 \boldsymbol{a}_{c, p}+\boldsymbol{a}_{d, p}\right)} \\
\boldsymbol{n} \times\left.\boldsymbol{h}_{p}\right|_{\gamma_{p}^{+}}=\frac{1}{2}\left[\sigma \beta \partial_{t}\left(2 \boldsymbol{a}_{c, p}+\boldsymbol{a}_{d, p}\right)+\frac{1}{\mu \beta} \boldsymbol{a}_{d, p}\right]-\boldsymbol{n} \times\left.\boldsymbol{h}_{u}\right|_{\gamma_{p}^{+}} \\
=\frac{1}{2}\left[\sigma \beta \partial_{t}\left(2 \boldsymbol{a}_{c, p}+\boldsymbol{a}_{d, p}\right)+\frac{1}{\mu \beta} \boldsymbol{a}_{d}\right]-\boldsymbol{k}_{f, p}
\end{gathered}
$$

where $\boldsymbol{a}_{d, p}$ and $\mathbf{a}_{c, p}$ are respectively the discontinuous and continuous components of the field $\boldsymbol{a}_{p}$. It should be noted that $\boldsymbol{a}_{d, p}$ is considered as zero on the negative side $\Gamma_{t s, p}^{-}$of the TS, which cancels the magnetic flux entering there [3]. $d$ (the thickness of the TS), $\delta$ (the skin depth) and $\beta$ are also given [3]. The discontinuity $\left[-\boldsymbol{n} \times\left.\boldsymbol{h}_{u}\right|_{\gamma_{p}^{+}}\right.$in (6) is really an SS for the volume correction.

\section{SEQUENCE OF WEAK FORMULATIONS}

\section{A. b-Conformal Formulation}

The weak $\boldsymbol{b}_{p}$-conformal formulation for problem $p$ is achieved from the weak form of the Ampere's law (1a), i.e. [47]:

$$
\begin{gathered}
\left(\mu_{p}^{-1} \operatorname{curl} \boldsymbol{a}_{p}, \operatorname{curl} \boldsymbol{a}_{p}^{\prime}\right)_{\Omega_{i}}+\left(\sigma_{p} \partial_{t} \boldsymbol{a}_{p}, \boldsymbol{a}_{p}^{\prime}\right)_{\mathrm{p}}+\left(\sigma_{p} \operatorname{grad} v_{p}, \boldsymbol{a}_{p}^{\prime}\right)_{\Omega_{c, p}}+ \\
\left(\boldsymbol{h}_{s, p}, \operatorname{curl} \boldsymbol{a}_{p}^{\prime}\right)_{\Omega_{c, p}}+\left(\boldsymbol{j}_{s, p}, \operatorname{curl} \boldsymbol{a}_{p}^{\prime}\right)_{\Omega_{c, p}}+\left\langle\boldsymbol{n} \times \boldsymbol{h}_{p}, \boldsymbol{a}_{p}^{\prime}\right\rangle_{\Gamma_{h, p}-\gamma_{p}}+ \\
\left\langle\boldsymbol{n} \times \boldsymbol{h}_{p}, \boldsymbol{a}_{p}^{\prime}\right\rangle_{\Gamma_{b, p}}+\left\langle-\left[\boldsymbol{n} \times \boldsymbol{h}_{p}\right]_{\gamma_{p}}, \boldsymbol{a}_{p}^{\prime}\right\rangle_{\gamma_{p}}=\left(\boldsymbol{j}_{s}, \boldsymbol{a}_{p}^{\prime}\right)_{\Omega_{s, p}} \quad(7) \\
\forall \boldsymbol{a}_{p}^{\prime} \in H_{p}^{1}\left(\Omega_{p}\right)
\end{gathered}
$$

where $H_{p}^{1}\left(\Omega_{p}\right)$ is a gauged curl-conform function space presented in $\Omega_{p}$, containing the basis functions for $\boldsymbol{a}_{p}$ and for the test function $\boldsymbol{a}_{p}^{\prime}$. Factors $(., .)_{\Omega_{i}}$ and $\langle., .\rangle_{\Gamma_{i}}$ are respectively notations of a volume intergal in $\Omega_{i}$ and a surface intergal on $\Gamma_{i}$ of the product of their vector field arguments. The surface integral term on $\Gamma_{h, p}$ accounts for natural BCs of type (3 b), which is usually zero. The term $\left\langle-\left[\boldsymbol{n} \times \boldsymbol{h}_{p}\right]_{\gamma_{p}}, \boldsymbol{a}_{p}^{\prime}\right\rangle_{\gamma_{p}}$ in (9) is the TS model and can be expressed as [3]:

$$
\begin{aligned}
& \left\langle\left[\boldsymbol{n} \times \boldsymbol{h}_{p}\right]_{\gamma_{p}}, \boldsymbol{a}_{p}^{\prime}\right\rangle_{\gamma_{p}}=\left\langle\left[\boldsymbol{n} \times \boldsymbol{h}_{p}\right]_{\gamma_{p}}, \boldsymbol{a}_{c, p}^{\prime}\right\rangle_{\gamma_{p}} \\
+ & \left\langle\boldsymbol{n} \times\left.\boldsymbol{h}_{p}\right|_{\gamma_{p}^{+}}, \boldsymbol{a}_{d, p}^{\prime}\right\rangle_{\gamma_{p}^{+}}+\left\langle\boldsymbol{n} \times\left.\boldsymbol{h}_{p}\right|_{\gamma_{p}^{-}}, \boldsymbol{a}_{d, p}^{\prime}\right\rangle_{\gamma_{p}^{-}}
\end{aligned}
$$

where $\boldsymbol{a}_{d, p}^{\prime}$ and $\boldsymbol{a}_{c, p}^{\prime}$ are the test functions and $\boldsymbol{a}_{d, p}^{\prime}$ is equal to zero on the negative side $\Gamma_{t, p}^{-}=\gamma_{t, p}^{-}$of the TS [3]. The terms $\left\langle\left[\boldsymbol{n} \times \boldsymbol{h}_{p}\right]_{\gamma_{p}}, \boldsymbol{a}_{c, p}^{\prime}\right\rangle_{\gamma_{p}}$ and $\left\langle\boldsymbol{n} \times\left.\boldsymbol{h}_{p}\right|_{\gamma_{p}^{+}}, \boldsymbol{a}_{d, p}^{\prime}\right\rangle_{\gamma_{p}^{+}}$are already defined in (5) and (6). Thus, (8) becomes:

$$
\begin{aligned}
& \left\langle[\boldsymbol{n} \times \boldsymbol{h}]_{\gamma_{p}}, \boldsymbol{a}_{p}^{\prime}\right\rangle_{\gamma_{p}}=-\left\langle\sigma \beta \partial_{t}\left(2 \boldsymbol{a}_{c, p}+\boldsymbol{a}_{d, p}\right), \boldsymbol{a}_{c, p}^{\prime}\right\rangle_{\gamma_{p}}+ \\
& \frac{1}{2}\left\langle\left[\sigma \beta \partial_{t}\left(2 \boldsymbol{a}_{c, p}+\boldsymbol{a}_{d, p}\right)+\frac{1}{\mu \beta} \boldsymbol{a}_{d}\right], \boldsymbol{a}_{c, p}^{\prime}\right\rangle_{\gamma_{p}}-\boldsymbol{k}_{f, p}
\end{aligned}
$$

\section{B. Volume Correction and Robust Correction Procedure} Replacing TS Representation

The TS solution is obtained by combining (7), (8) and (9). The solution is now improved by the volume correction (e.g. problem $k$ ) via VSs $\left(\boldsymbol{j}_{s, p}, \operatorname{curl} \boldsymbol{a}_{p}^{\prime}\right)_{\Omega_{c, p}}\left(\boldsymbol{h}_{s, p}, \operatorname{curl} \boldsymbol{a}_{p}^{\prime}\right)_{\Omega_{c, p}}$ given by $(4 a)$ and $(4 b)$. This means that these fields need to be transferred from the mesh of the TS problem $p$ to the mesh of next problem $k$ (volume correction $k$ ) via a projection method [9]. For that, the weak form for the problem $k$ is written as

$$
\begin{gathered}
\left(\mu_{k}^{-1} \operatorname{curl} \boldsymbol{a}_{k}, \operatorname{curl} \boldsymbol{a}_{k}^{\prime}\right)_{\Omega_{k}}+\left(\sigma_{k} \partial_{t} \boldsymbol{a}_{k}, \boldsymbol{a}_{k}^{\prime}\right)_{\Omega_{c, k}} \\
+\left(\sigma_{k} \operatorname{grad} v_{k}, \boldsymbol{a}_{k}^{\prime}\right)_{\Omega_{c, k}}+\left(\left(\mu_{k}^{-1}-\mu_{p}^{-1}\right)\left(\operatorname{curl} \boldsymbol{a}_{p}, \operatorname{curl} \boldsymbol{a}_{k}^{\prime}\right)_{\Omega_{k}}\right. \\
+\left\langle\left[\boldsymbol{n} \times \boldsymbol{h}_{k}\right]_{\gamma_{t, k},} \boldsymbol{a}_{k}^{\prime}\right\rangle_{\gamma_{t, k}}+\left(\sigma_{k} \partial_{t} \boldsymbol{a}_{p}, \boldsymbol{a}_{k}^{\prime}\right)_{\Omega_{c, k}}=0 \\
\forall \boldsymbol{a}_{k}^{\prime} \in H_{k}^{1}\left(\Omega_{k}\right)
\end{gathered}
$$

At the same time to the VSs in (10), the SSs related to ICs [4-5] have to extract the TS discontinuities. Thus, the trace $\left.\boldsymbol{n} \times \boldsymbol{h}_{k}\right]_{\gamma_{t, k}}$ in (10) is strongly fixed as:

$$
\left\langle\left[\boldsymbol{n} \times \boldsymbol{h}_{k}\right]_{\gamma_{t, k}}, \boldsymbol{a}_{k}^{\prime}\right\rangle_{\gamma_{t, k}}=-\left\langle\left[\boldsymbol{n} \times \boldsymbol{h}_{p}\right]_{\gamma_{t, k}}, \boldsymbol{a}_{k}^{\prime}\right\rangle_{\gamma_{t, k}}
$$

At the discrete level, the source field $\boldsymbol{a}_{p}$ solved in the mesh of the TS problem $p$ is projected to the mesh of problem $k$ in (10), with $\Omega_{c, k}$ limited to the volume correction, which thus reduces the computational effort of the process of projection. The volume correction in (10) is really sensitive to cancellation errors, with dramatic effects on the calculation of the field $\boldsymbol{a}_{p}$ (see Figure 5 in [6]). In order to avoid the cancellation error, we need to combine a problem $k$ - $a$ with:

$$
\boldsymbol{h}_{k-a}=\mu_{k-a}^{-1} \boldsymbol{b}_{k-a}+\left(\mu_{k-a}^{-1}-\mu_{p}^{-1}\right) \boldsymbol{b}_{p}
$$

Considering a perfect magnetic region in $\Omega_{c, k}\left(\mu_{k-a}=\right.$ $\infty, \mu_{k-a}^{-1}=0$ ), and a problem $k-b$ with:

$$
\boldsymbol{h}_{k-b}=\mu_{k-b}^{-1} \boldsymbol{b}_{k-b}+\left(\mu_{k-b}^{-1}-\mu_{k-a}^{-1}\right)\left(\boldsymbol{b}_{k-a}+\boldsymbol{b}_{p}\right)
$$

presenting a change to the actual permeability $\left(\mu_{k-b}=\right.$ $\mu_{\text {acutal }}=\mu_{k}$ ). The problem $k$ - $a$ uses a SS only contributing on the positive side of $\Gamma_{c, k}^{+}$of $\Gamma_{c, k}$, that is: 


$$
\left[\boldsymbol{n} \times \boldsymbol{h}_{k-a}\right]_{\Gamma_{c, k}}=\boldsymbol{n} \times\left.\boldsymbol{h}_{k-a}\right|_{\Gamma_{c, k}^{+}}=-\boldsymbol{n} \times\left.\boldsymbol{h}_{p}\right|_{\Gamma_{c, k}^{+}}=\boldsymbol{j}_{s u r, k-a}
$$

with $\boldsymbol{h}_{s, k-a}=0$ and $\boldsymbol{b}_{k-a} \neq 0$. The trace $\boldsymbol{n} \times\left.\boldsymbol{h}_{p}\right|_{\Gamma_{c, k}^{+}}$is originally presented in (18) for the problem $p$ limited to $\Gamma_{c, k}=$ $=\Gamma_{t, p}$. It maybe also naturally presented via the volume in (10), that is:

$$
\begin{gathered}
\left\langle\left[\boldsymbol{n} \times \boldsymbol{h}_{k-a}\right]_{\Gamma_{c, k}}, \boldsymbol{a}_{k}^{\prime}\right\rangle_{\Gamma_{c, k}}=-\left\langle\boldsymbol{n} \times\left.\boldsymbol{h}_{p}\right|_{\Gamma_{c, k}^{+}}, \boldsymbol{a}_{k}^{\prime}\right\rangle_{\Gamma_{c, k}}= \\
\left(\mu_{p}^{-1} \operatorname{curl} \boldsymbol{a}_{p},\left.\operatorname{curl} \boldsymbol{a}_{k}^{\prime}\right|_{\Gamma_{c, k}^{+}}\right)_{\Omega_{\mathrm{TL}}}(15)
\end{gathered}
$$

where $\Omega_{\mathrm{TL}}$ is limited to one single layer of FEs touching $\Gamma_{c, k}^{+}$, because it involves only the associated trace $\boldsymbol{n} \times\left.\boldsymbol{a}_{k}^{\prime}\right|_{\Gamma_{c, k}^{+}}$[5] ( $\boldsymbol{a}_{k-a}^{\prime}=\boldsymbol{a}_{k-b}^{\prime}=\boldsymbol{a}_{k}^{\prime}$ because of the same property on the mesh). For the problem $k-b$, the VS has:

$$
\boldsymbol{h}_{s, k-b}=\left(\mu_{k-b}^{-1}-\mu_{k-a}^{-1}\right)\left(\boldsymbol{b}_{k-a}+\boldsymbol{b}_{p}\right)
$$

with $\mu_{k-b}=\mu_{\text {acutal }}=\mu_{k}$ and $\mu_{k-a}^{-1}=0$. Both problems $k-a$ and $k \_b$ are being solved at the same time, with the SS $\boldsymbol{j}_{f, k}=\boldsymbol{n} \times\left.\boldsymbol{h}_{p}\right|_{\Gamma_{c, k}^{+}}=\boldsymbol{j}_{f, k-a}$ and the resulting relation is:

$$
\begin{gathered}
\boldsymbol{h}_{k}=\boldsymbol{h}_{k-a}+\boldsymbol{h}_{k-b}= \\
-\mu_{p}^{-1} \boldsymbol{b}_{p}+\mu_{k-b}^{-1} \boldsymbol{b}_{k-b}+\mu_{k-b}^{-1}\left(\boldsymbol{b}_{k-a}+\boldsymbol{b}_{p}\right)= \\
=\mu_{k}^{-1}\left(\boldsymbol{b}_{k-a}+\boldsymbol{b}_{p}\right)+\left(\mu_{k}^{-1}-\mu_{p}^{-1}\right) \boldsymbol{b}_{p}
\end{gathered}
$$

This procedure needs the projection of the source field in the added magnetic region (for VSs) as well as in the layer of FEs surrounding this region (for $\mathrm{SSs}$ ).

\section{Projections of Solutions between Meshes}

In the SPM strategy, the TS is considered as an SS in a submodel $\Omega_{c, k}$ which is a subset of $\Omega_{k}$. At the discrete level, the source field from the previous problem (e.g. $\boldsymbol{a}_{p}$ in the previous mesh problem $p$ ) is projected in the mesh of the current (new) problem (e.g. problem $k$ ). This can be done via a projection method [8] of its curl limited to $\Omega_{c, k}$, i.e.:

$$
\begin{gathered}
\left(\operatorname{curl} \boldsymbol{a}_{p, k-p r o j}, \operatorname{curl} \boldsymbol{a}_{k}^{\prime}\right)_{\Omega_{c, k}}=\left(\operatorname{curl} \boldsymbol{a}_{p}, \operatorname{curl} \boldsymbol{a}_{k}^{\prime}\right)_{\Omega_{c, k}}, \\
\forall \boldsymbol{a}_{k}^{\prime} \in \boldsymbol{H}_{k}^{1}\left(\operatorname{Curl}, \Omega_{k}\right)
\end{gathered}
$$

where $\boldsymbol{H}_{k}^{1}\left(\mathrm{Curl}, \Omega_{c, k}\right)$ is a gauged curl-conform function space for the $k$-projected source $\boldsymbol{a}_{p, k-p r o j}$ and the test function $\boldsymbol{a}_{k}^{\prime}$.

\section{NUMBERICAL TEST}

The numerical test is an actual problem including an inductor located below a shielding thin plate (Figure 1).

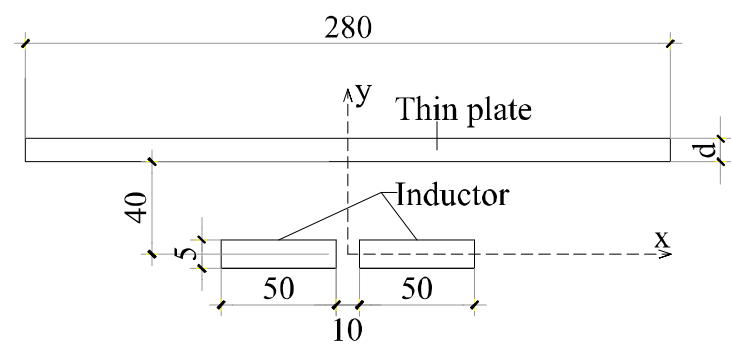

Fig. 1. Flux geometry of inductor and thin plate (dimensions in $\mathrm{mm}$ )
The inductor carries a fixed current density (excitation current $I=1 \mathrm{~A}$, frequency $f=50 \mathrm{~Hz}$, number of turns $N=1000$ ).

The test problem is solved in three steps:

- Step 1: The stranded inductors with the TS model approximations ( $\mathrm{SP} p$ ) are first solved

- Step 2: The volume correction (SP $k$ ) is given to overcome the TS approximations at step 1

- Step 3: The robust correction procedure is considered to improve cancelation errors at step 2

The TS solution SP $p$ on the magnetic vector potential at step 1 considered with the thin plate and the stranded inductor is shown in Figure 2(a). Next, a volume correction SP $k$ then replaces the TS approximation with an actual volume covering the plates and their surroundings without including the stranded inductor anymore (Figure 2(b)). As presented, this volume correction is very sensitive to cancellation errors, with dramatic effects on the calculation, thus it is improved by a robust correction procedure (Figure 2(c)).

(a)

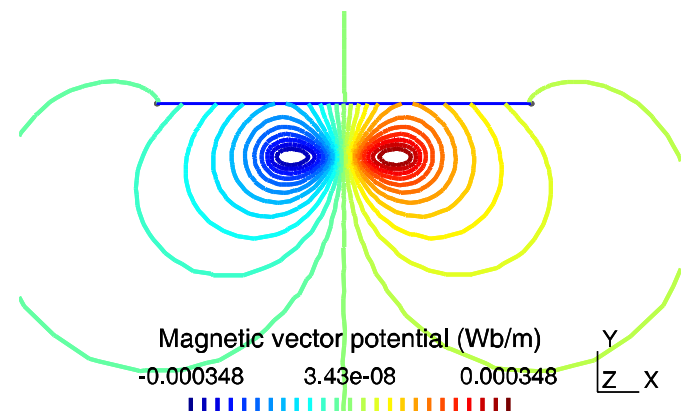

| | | | | | | | | | | | | | | | | | | | | | | | | |

(b)

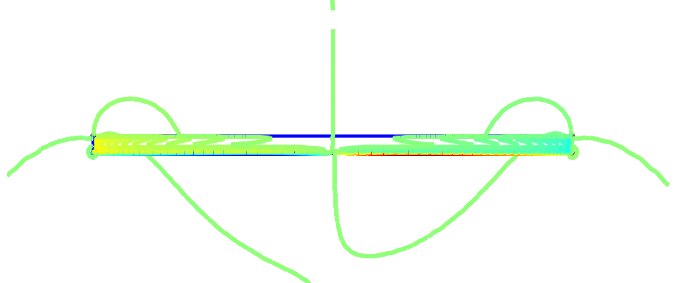

Volume correction with cancellation error $(\mathrm{Wb} / \mathrm{lm}$ ) $\begin{array}{lll}-0.000143 & -2.36 e-09 & 0.000143 \quad Z \quad Z X\end{array}$

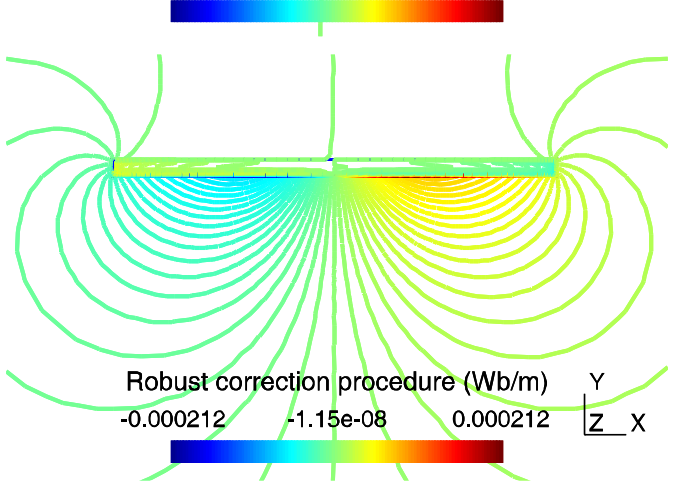

Fig. 2. Flux lines (real part) on the magnetic vector potential for (a) the TS solution, (b) volume correction with cancellation errors, and (c) robust correction procedures (c) $\left(d=10 \mathrm{~mm}, f=50 \mathrm{~Hz}, \mu_{r}=100\right.$ and $\left.\sigma=59 \mathrm{MS} / \mathrm{m}\right)$. 
The relative robust correction procedure of the longitudinal magnetic flux along the thin plate increases with the different thicknesses $\left(f=50 \mathrm{~Hz}, \mu_{r}=100\right.$ and $\left.\sigma=59 \mathrm{MS} / \mathrm{m}\right)$, as shown in Figure 3. For thickness $d=10 \mathrm{~mm}$, it can reach several tens percent in the vicinity near edges and corners of the plate, i.e. $70 \%$, with $f=50 \mathrm{~Hz}, \mu_{r}=100, \sigma=59 \mathrm{MS} / \mathrm{m}$ and $\delta=1.977 \mathrm{~mm}$. For the lower thickness $d=2.5 \mathrm{~mm}$, the error is lower than $15 \%$ from the middle to the end.

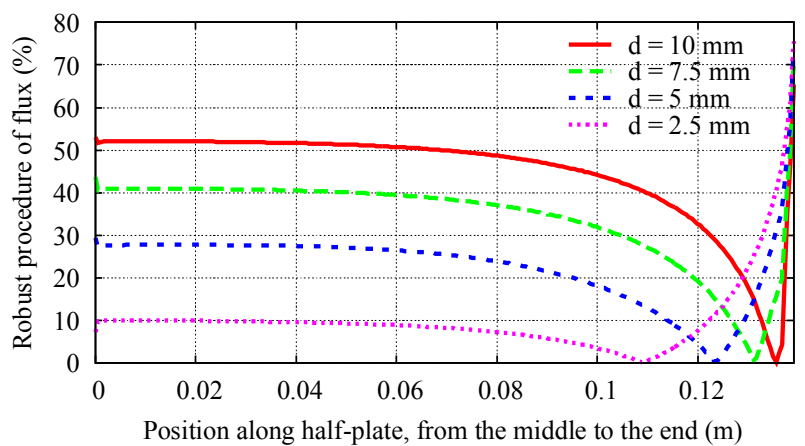

Fig. 3. Relative robust correction procedure of the longitudinal magnetic flux along the thin plate, with different thicknesses $\left(f=50 \mathrm{~Hz}, \mu_{r}=100\right.$ and $\sigma=59 \mathrm{MS} / \mathrm{m})$.

(a)

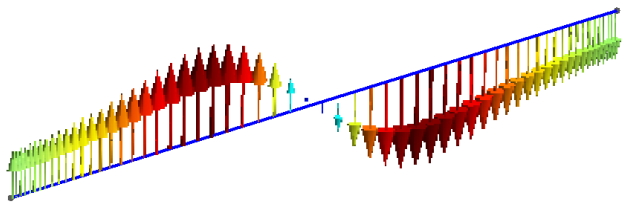

(b)
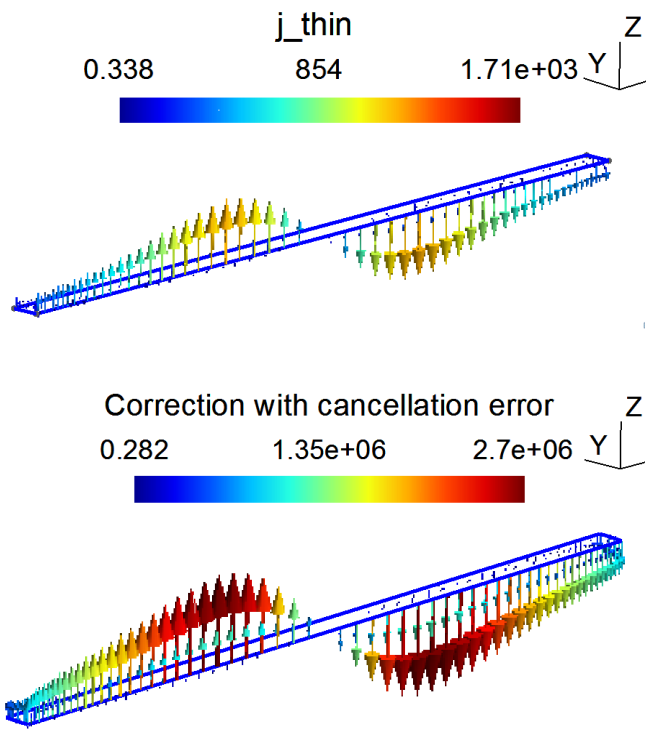

(c)

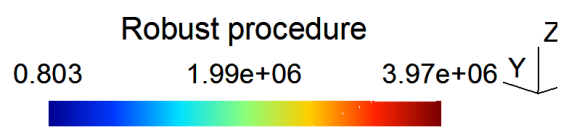

Fig. 4. Distribution of eddy current densities with (a) the TS solution, (b) cancellation error, and (c) the robust procedure $\left(d=10 \mathrm{~mm}, f=50 \mathrm{~Hz}, \mu_{r}=100\right.$ and $\sigma=59 \mathrm{MS} / \mathrm{m})$.

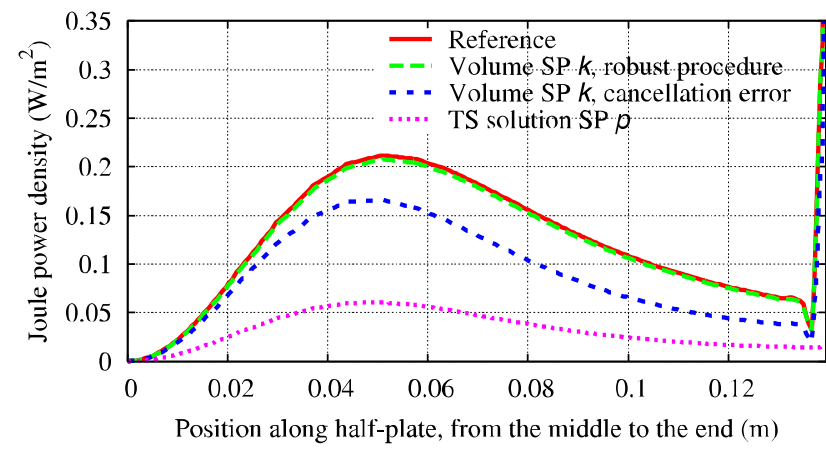

Fig. 5. Joule power loss densities with TS solution, volume correction with cancellation error, and the robust procedure along the thin plate $\left(d=10 \mathrm{~mm}, f=50 \mathrm{~Hz}, \mu_{r}=100\right.$ and $\left.\sigma=59 \mathrm{MS} / \mathrm{m}\right)$.

The eddy current density of the TS SP $p$ (Figure 4(a)) is corrected by the volume correction SP $k$ (Figure 4(b)) without taking the cancellation error into account. The robust correction procedure (Figure 4(c)) is proposed to improve the cancellation errors to overcome the volume correction at step 2. The error obtained is $32 \%$ at the plate end. The inaccuracy on the joule power loss density along the half plate of the TS solution approximation SP $p$, the volume correction SP $k$ and the robust correction procedure are pointed out in Figure 5, with $d=10$ $\mathrm{mm}, f=50 \mathrm{~Hz}, \mu_{r}=100, \sigma=59 \mathrm{MS} / \mathrm{m}$. The significant errors approximately reach $58 \%$ between the TS solution SP $p$ and the volume correction SP $k$, or nearly $20 \%$ between the volume correction SP $k$ and the robust correction procedure. The robust correction procedure is then compared with the reference solution computed from the traditional Finite Element Method (FEM) $[7,8,10]$. This is an agreement to illustrate a very suitable validation of the robust correction procedure developed in the perturbation technique.

\section{DISCUSSION AND CONCLUSIONS}

Robust correction procedure with the magnetic vector potential formulation has been sucessfully developed via a perturbation technique in order to improve the cancellation errors on the local fields of magnetic vector potential, magnetic flux, eddy current loss, and Joule power loss density inherent to the TS approximation and the volume correction. The computed results of the extend method are close to the reference FEM calculation solution $[7,8,10]$, which is a very good validation of the studied technique. The development has been successfully done with the linear case in the frequency domain. Extension of the method could be further performed in two-way coupling [12]. All the steps of the technique have been validated and applied to the practical problem.

\section{REFERENCES}

[1] H. Tsuboi, T. Asahara, F. Kobaysashi, T. Misaki, "Eddy current analysis on thin conducting plate by an integral equation method using edge elements", IEEE Transactions on Magnetics, Vol. 33, No. 2, pp. 13461349, 1997

[2] C. Geuzaine, P. Dular, W. Legros, "Dual formulations for the modeling of thin electromagnetic shells using edge elements", IEEE Transactions on Magnetics, Vol. 36, No. 4, pp. 799-802, 2000

[3] P. Dular, V. Q. Dang, R. V. Sabariego, L. Krahenbuhl, C. Geuzaine, "Correction of thin shell finite element magnetic models via a 
subproblem method", IEEE Transactions on Magnetics, Vol. 47, No. 5, pp. 1158--1161, 2011.

[4] V. Q. Dang, P. Dular, R. V. Sabariego, L. Krahenbuhl, C. Geuzaine, "Subproblem approach for thin shell dual finite element formulations", IEEE Transactions on Magnetics, Vol. 48, No. 2, pp. 407-410, 2012

[5] P. Dular, R. V. Sabariego, "A perturbation method for computing field distortions due to conductive regions with h-conform magnetodynamic finite element formulations", IEEE Transactions on Magnetics, Vol. 43, No. 4, pp. 1293-1296, 2007

[6] P. Dular, R. V. Sabariego, C. Geuzaine, M. V. Ferreira da Luz, P. KuoPeng, L. Krahenbuhl, "Finite element magnetic models via a coupling of subproblem of lower dimensions", IEEE Transactions on Magnetics, Vol. 46, No. 8, pp. 2827-2380, 2010.

[7] S. Koruglu, P. Sergeant, R.V. Sabarieqo, V. Q. Dang, M. De Wulf "Influence of contact resistance on shielding efficiency of shielding gutters for high-voltage cables", IET Electric Power Applications, Vol. 5, No.9, pp. 715-720, 2011

[8] G. Meunier, The finite element method for electromagnetic modeling, John Wiley \& Sons, 2008

[9] C. Geuzaine, B. Meys, F. Hernotte, P. Dular, W. Legros, “A Galerkin projection method for mixed finite elements", IEEE Transactions on Magnetics, Vol. 35, No. 3, pp. 1483-1441, 1999

[10] K. Abubakri, H. Veladi, "Investigation of the behavior of steel shear walls using finite elements analysis", Engineering, Technology \& Applied Science Research, Vol. 6, No. 5, pp. 1155-1157, 2016

[11] F. G. Kizilcay Abdulla, R. Abdulla, "A comparative application for evaluating composite fabrics used in electromagnetic shielding", Engineering, Technology \& Applied Science Research, Vol. 7, No. 6, pp. $2156-2159,2017$

[12] V. D. Quoc, C. Geuzaine, "Two-way coupling of thin shell finite element magnetic models via an iterative subproblem method", COMPEL - The International Journal for Computation and Mathematics in Electrical and Electronic Engineering, ahead-of-print, doi: 10.1108/COMPEL-01-2020-0035, 2020

\section{AUTHOR PROFILE}

Vuong Dang Quoc received his PhD degree in 2013 from the Faculty of Applied Sciences at the University of Liege in Belgium. After that he came back to the Hanoi University of Science and Technology in September 2013, where he is currently working as the director of the Training Center of Electrical Engineering, School of Electrical Engineering, Hanoi University of Science and Technology. Dr. Vuong Dang Quoc's research domain encompasses modeling of electromagnetic systems by coupling of subproblem method with application to thin shell models. 